Jiafu Su, Yu Yang*, Kunpeng Yu and Na Zhang

\title{
A Method of Partner Selection for Knowledge Collaboration Teams using Weighted Social Network Analysis
}

DOI 10.1515/jisys-2016-0140

Received August 14, 2016; previously published online April 12, 2017.

\begin{abstract}
Partner selection is the primary aspect of the formation of knowledge collaboration teams (KCTs). We propose a method of partner selection for KCTs based on a weighted social network analysis (SNA) method in which the individual knowledge competence and the collaboration performance of candidates are both considered. To select the desired partners, a biobjective 0-1 model is built, integrating the knowledge competence and collaboration performance, which is an NP-hard problem. Then, a multiobjective genetic algorithm (MOGA) is developed to solve the proposed model. Finally, a real-world example is provided to illustrate the applicability of the model, and the MOGA is implemented to search for Pareto solutions of partner selection for KCT in this case. Moreover, some simulation examples are used to test the efficiency of the algorithm. The results suggest that the proposed method can support effective and practical partner selection.
\end{abstract}

Keywords: Collaboration performance, knowledge collaboration team, knowledge competence, partner selection, social network analysis.

\section{Introduction}

With the tendency of global economic integration and the uncertainty of market competition environment, firms increasingly tend to focus on the integration of internal and external knowledge resources to obtain the knowledge complementary advantages and reduce the risk of knowledge innovation by cooperating and interacting with other firms, research institutions, and university research units [6]. These changes have led to the development of knowledge collaboration mode. Aiming at collaborative knowledge innovation, the collaborative new product development (NPD) team, technology innovation team, cross-functional team, and collaborative product service team all can be called as the knowledge collaboration team (KCT) [16]. By adopting KCT, firms can work closely with their cooperative partners and obtain more new insights and knowledge to push forward collaboration and more open forms of innovation [40]. In several industries, such as complex products [44], pharmaceutical manufacturing [25], digital products [48], equipment for outdoor sports [38], and video games [27], KCTs have been widely applied to facilitate product and service innovations for future development. A few business examples include Lego's collaboration with customers on the development of its NXT Robot [39], Xiaomi's collaboration with manufacturers and customers on the design of smartphones [48], and Snap-on's collaboration with automotive technicians who create the logic for their computerized diagnostic systems [39]. Practices show that KCTs have been an emerging trend in management with their collaborative advantages.

Correction note: Correction added after online publication April 12, 2017: "Chongqing Key Laboratory of Electronic Commerce \& Supply Chain System, Chongqing Technology and Business University, Chongqing, China” was added to the affiliation of Jiafu Su in order to reflect a change of affiliations during the work on this article.

*Corresponding author: Yu Yang, Chongqing University - Industrial Engineering, Chongqing, China, e-mail: yuyang@cqu.edu.cn Jiafu Su: Chongqing Key Laboratory of Electronic Commerce \& Supply Chain System, Chongqing Technology and Business University, Chongqing, China; and The State Key Laboratory of Mechanical Transmission, Chongqing University, Chongqing, China Kunpeng Yu: Management College of Ocean University of China, Qingdao, China Na Zhang: The State Key Laboratory of Mechanical Transmission, Chongqing University, Chongqing, China 
KCTs consist of partners from different functional areas such as engineering, manufacturing, or marketing or from various knowledge domains such as chemistry, electronics, or biomaterials [40]. In KCTs, team partners have different knowledge and ideas for the specific task [16]. Moreover, each team partner requires collaborative interactions with other partners to stimulate the synergy of KCT [17]. In the development of KCTs, team formation and partner selection is the first stage. To ensure the success of KCTs, it is important to choose the team partners with desirable qualifications to effectively carry out plans and produce successful projects. Recently, partner selection for KCTs has attracted increasing concerns [12, 28]. The existing researches mainly focus on the individual performance of candidates, while they seldom incorporate the collaborative performance between candidates in partner selection for KCTs. In practice, except for the individual performance of partners, the collaboration among partners of KCT also plays a vital role as the performance of a KCT depends on the good combination of knowledge and expertise of all team partners [28]. Thus, it is very necessary to consider the individual performance and collaboration performance among partners in partner selection for KCTs. In this consideration, the aim of this paper is to propose a novel approach of partner selection for KCTs using the individual knowledge competence and collaboration performance of the candidates. In this way, this paper tries to shed some light on the partner selection problem of KCTs with a new angle and method to help decision-makers to select desired partners with comprehensive advantages.

The reminder of the paper is organized as follows. Section 2 reviews the related work on partner selection for teams. In Section 3, the weighted SNA method is proposed to assess the knowledge competence and collaboration performance of candidates and presents a multiobjective model of partner selection for KCTs. In Section 4, a multiobjective genetic algorithm (MOGA) is developed to solve the model. Section 5 demonstrates the application of the model and the algorithm in a real-world case of KCT. Finally, the main contributions and future research of this work are summarized in Section 6.

\section{Literature Review}

Partner selection is a primary decision problem in the formation stage of KCTs, which has great significance for the success of collaborative innovation. In the existing researches of partner selection, a number of scholars have focused on the characteristics and qualifications of desired partners. Chen and Lin [8] discussed the main requirements for desired partners, such as multiple functional knowledge domains, teamwork skills, and working relationship. Zhang and Zhang [47] regarded expertise and experience, learning and knowledge sharing, communication, problem-solving ability, and personality characteristics as the main attributes for partner selection. To obtain the complementary advantages, Haque et al. [22] stated that good teams should consist of specialized partners with different values, interests, and knowledge domains. Knowledge innovation is a knowledge-intensive activity [14], and the above works all note that the knowledge competence plays a vital role for partner selection.

On the contrary, collaborative performance is also very important for partner selection for KCTs. In practice, collaborative knowledge innovations need the efforts of partners with a variety of knowledge and skills. The good collaboration within KCTs is necessary to promote mutual understanding, interpersonal interaction, and cohesiveness, to reduce uncertainty and conflicts, and to improve innovation performance [11]. Wi et al. [43] stated that partners with good collaboration can integrate more resources in collaborative teams. Moreover, some researchers argued that prior good collaboration experience will encourage firms to choose past cooperators as future partners [19, 29]. An empirical study in North America showed that the longer and better the prior collaborations between two firms are, the more likely they will recreate their collaborations [21]. Fan et al. [15] stated that partners with good collaboration history always are the priority, and prior collaboration information should be made a valuable reference for the formation of collaborative teams. Therefore, not only the knowledge competence of the individual candidate but also the collaboration information among candidates should be considered in partner selection for KCTs. Also, a comprehensive and effective approach is needed to model and evaluate the above attributions.

The model method for selecting partners can be divided into two categories: model for forecasting candidates' performance by means of historical data and model for selecting persons who meet the requirements 
of projects. Huang et al. [24] proposed a model to forecast potential partners' performance using the personal data, such as age, service years, number of projects, and performance. Chien and Chen [10] forecasted employees' performance, turnover, and job quitting possibility with eight independent variables: age, gender, marital status, school, major, degree, work experience, and the recruitment channels. In these models, the forecasts mostly rely on managers' subjective evaluation. On the contrary, there are more academic efforts to select qualified partners required for a specific project. Wi et al. [43] used the fuzzy inference system and social network analysis (SNA) method to evaluate candidates' knowledge and collaboration competence for a specific project. Fan et al. [15] proposed a comprehensive model integrating individual and collaborative attributions to select desired partners with specific qualifications required by R\&D teams. Based on the Myers-Briggs type indicator, Zhang and Zhang [47] proposed a multiobjective optimization model for NPD team formation considering the comprehensive capabilities and interpersonal relationships of the candidates. Selecting the qualified partners for specific projects aims at the realities of the project and its candidates, which has a more realistic significance than the forecasting model, and becomes the research mainstream of partner selection.

In the related literature on partner selection, we find that the analytic hierarchy process (AHP) and fuzzy methods are generally used to evaluate candidates' qualifications [23, 49]. However, in the fuzzy methods, it is difficult to obtain the fuzzy membership functions because the lower and upper bounds of the functions are uncertain. Moreover, in the AHP method, a lot of time is used to make $n(n-1) / 2$ pairwise comparisons with $n$ criteria, and it is difficult to assure the consistency. In addition, some researchers tried to use other methods to quantitatively assess the comprehensive capabilities of candidates. For instance, Zhang and Zhang [47] implemented a fuzzy-AHP method based on fuzzy linguistic preference relations to evaluate team members' capabilities. Based on the social network relationships, Feng and Fan [16] proposed a collaborative matrix method to quantify collaborative performance among candidates. Wang et al. [42] established a multilevel extendible comprehensive evaluation method to evaluate the candidates' individual capacity, coordination ability, and match adaptation among candidates. These examples indicate that the above methods can effectively assess and evaluate the candidates' qualifications in the specific cases, which provide valuable enlightenment to our study.

In practice, there are formal and informal collaborative relationships among candidates. Moreover, the candidates and the collaborative relationships among candidates have objectively formed a collaborative social network [16, 32, 42]. In the context of social networks, the SNA method can effectively investigate the characteristics of network nodes and network edges among nodes. Therefore, the researchers increasingly used the SNA method to analyze the collaborative organizations and to solve the practical problems of collaborative organizations. Pereira and Soares [37] used the measures of SNA, such as density, degree centrality, and closeness centrality, to analyze the collaboration relationships. Wi et al. [43] used SNA to investigate the familiarity degree among candidates in collaborative teams. Li et al. [32] proposed a weighted social network model to evaluate the collaboration strength among members in a collaborative NPD team. In the context of KCTs, the SNA method provides us a new sight and methodology to investigate candidates' knowledge and collaboration qualifications. The knowledge and collaboration qualifications including knowledge stock, communication information, and project cooperation can be easily integrated and analyzed using the SNA method. Based on the above analysis, we developed a weighted SNA method for the assessment of the knowledge competence and collaboration performance of candidates. Also, based on the weighted SNA method, we propose a multiobjective optimization model of partner selection for KCTs, which addresses the knowledge competence and collaboration performance of candidates in this paper.

\section{Model of Partner Selection for KCTs}

\subsection{Problem Statement}

This paper focuses on the problem of partner selection for KCTs, whose candidates are from different organizations and with different knowledge backgrounds, using the attributions of individual knowledge competence 
and collaboration performance. In doing so, we developed a weighted social network to integrate all the candidates and their collaborative relationships. Also, the quantitative methods of knowledge competence and collaboration performance are proposed based on the SNA method. Furthermore, a multiobjective model of partner selection for KCTs is further proposed considering the above-quantified attributions.

The problem of partner selection for KCTs can be described as follows: Suppose that a KCT needs to select $m$ partners from a set of $n$ candidates, $P=\left\{P_{1}, P_{2}, \ldots, P_{n}\right\}$, where $P_{i}$ represents the $i$ th candidate. The knowledge competence of candidate $P_{i}$ is denoted as $K C_{i}$. Let $C P_{i j}$ be the collaborative performance between candidates $P_{i}$ and $P_{i}$. To choose the appropriate partners and form the view of knowledge and collaboration, managers should select $m$ partners with the optimal sum of the knowledge competence $K C_{i}$, and among the $m$ partners, there should be the optimal collaborative performance $C P_{i j}{ }^{*}$

\subsection{Weighted SNA to Assess Knowledge Competence and Collaboration Performance}

The SNA method conceptualizes social structure as a network with ties connecting actors and resources, which is an interdisciplinary methodology focusing on how structural regularities influence actors' behavior [7]. The social network perspective views any system as a set of interrelated nodes connected with ties. The nodes can represent entities at various levels, such as persons, firms, and countries. The ties among actors can be of many different types, such as friendship, collaboration, and competition, and can be characterized by multiple dimensions, such as duration, frequency, and closeness [35]. The typology in Figure 1 divides the dyadic relations into four basic types, i.e. similarities, social relations, interactions, and flows. Much of the social network researches can be seen as working out how these different kinds of ties affect each other.

In this work, we regard the candidate collectivity and the collaborative relationships among them as a social network, within, the nodes represent the candidates, and the ties are the collaborative relationships among candidates, such as paper and project collaboration. The candidate network can be denoted by an undirected weighted network $G=(P, E, W)$, with $n$ nodes as shown in Figure 2, and in which $P=\left\{P_{1}, P_{2}, \ldots, P_{n}\right\}$ is the set of candidates, $E=\left\{e=\left(P_{i}, P_{j}\right)\right\}$ represents the set of collaborative ties $e=\left(P_{i}, P_{j}\right)$ between candidates $P_{i}$ and $P_{j}$, and $W=\left\{w_{i}, w_{i j}\right\}$ denotes the weight sets of candidates and their ties.

\subsubsection{Node Weight of Candidate}

In this work, the node weight of candidate is used to reflect the individual knowledge competence. In this work, a decision matrix is built to evaluate the individual knowledge competence. Let $T=\left[t_{i g}\right]_{n \times l}$ be the decision matrix, where $t_{i g}$ is the consequence with a numerical value of candidate $P_{i}$ with respect to criterion $R_{g}$, $g=1,2,3, \ldots, l$. In practice, the criterion $R_{g}$ can be objective or subjective. If criterion $R_{g}$ is objective, the criterion value can be obtained by the statistic data or other objective data source. If criterion $R_{g}$ is subjective, the criterion value can be given by experts' assessment or AHP. Furthermore, regarding the commensurabil-

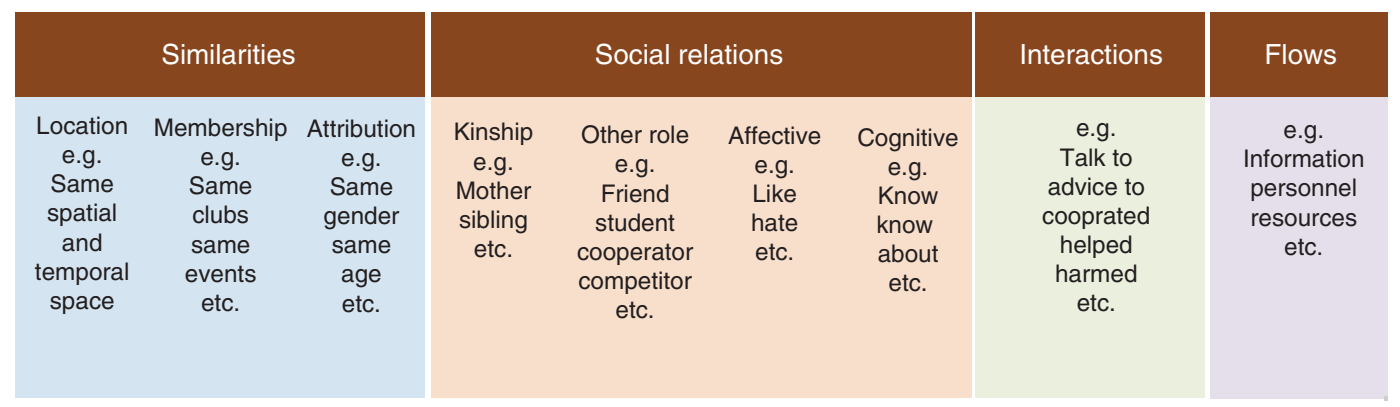

Figure 1: Typology of Types of Ties Among Persons in Social Networks. 


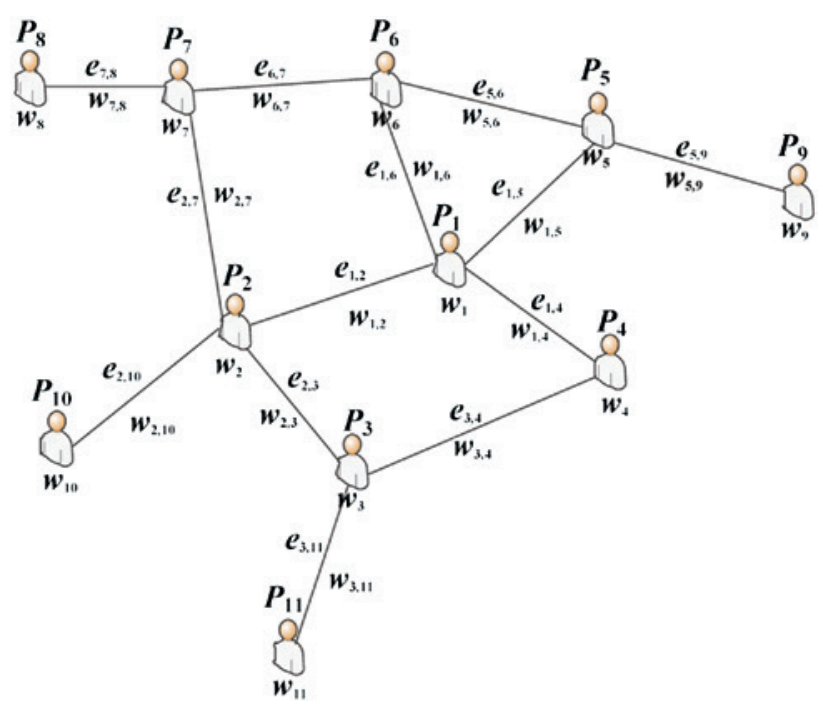

Figure 2: Example of Undirected Weighted Network of Candidates.

ity between various criteria, every element in matrix $T=\left[t_{i g}\right]_{n \times l}$ should be normalized into a corresponding element in matrix $T^{\prime}=\left[t_{i g}^{\prime}\right]_{n \times l}$ using the approach proposed by Yoon and Hwang [46].

$$
\begin{aligned}
& t_{i g}^{\prime}=\frac{t_{g}^{\max }-t_{i g}}{t_{g}^{\max }-t_{g}^{\min }} \\
& g=1,2,3, \cdots, l, \text { for cost criteria. } \\
& t_{i g}^{\prime}=\frac{t_{i g}-t_{g}^{\min }}{t_{g}^{\max }-t_{g}^{\min }} \\
& g=1,2,3, \cdots, l \text {, for benefit criteria. }
\end{aligned}
$$

where $t_{g}^{\max }=\max \left\{t_{i g} \mid i=1,2, \cdots, n\right\}, t_{g}^{\min }=\min \left\{t_{i g} \mid i=1,2, \cdots, n\right\}$.

Next, each criterion is given weight $v_{g}, \sum_{g=1}^{l} v_{g}=1, g=1,2, \cdots, l$, by direct assignment or AHP. Then, the individual knowledge competence $w_{i}$, which is the node weight, can be calculated by

$$
w_{i}=\mathrm{KC}_{i}=\sum_{g=1}^{l} v_{g} \cdot t_{i g}^{\prime}, g=1,2, \cdots, l
$$

\subsubsection{Tie Weight of Candidates' Collaboration}

The tie weight denotes the collaboration performance among candidates. In the context of KCT, there are two types of collaborative relationship among team partners: formal and informal collaborative relationships. The formal collaborative relationship is the cooperative relationship on knowledge collaboration project or task. On the contrary, the informal collaborative relationship implies the social relationship among partners. Based on the above classifications, we attempt to investigate collaborative performance at both sides of the formal and informal collaborative relationships.

For the formal collaborative relationship, it typically occurs and accumulates among partners via project collaboration. Some scholars argued that people favor the partners with whom they have successful cooperation before, which can reduce uncertainty regarding potential members' capabilities and reliabilities [29, 34]. 
We assume that partners who cooperated many projects together will have better collaboration on average than those who have cooperated less projects together. To account for this, we add together the strengths of the ties derived from each of the projects cooperated by a particular pair of partners. Thus, if $\sigma_{i}^{k}$ is 1, candidate $P_{i}$ was a participant of project $k$ and 0 if otherwise. Then, weight $F w_{i j}$ representing the strength of the formal collaboration between $P_{i}$ and $P_{j}$ can be obtained [36]:

$$
F w_{i j}=\sum_{k} \frac{\sigma_{i}^{k} \sigma_{j}^{k}}{n_{k}-1}
$$

where $n_{k}$ is the number of participants of project $k$, and we explicitly excluded single-participant projects. (They do not contribute to the collaborative network, and their inclusion would make $F w_{i j}$ ill defined.) This measure is illustrated by a simple example in Figure 3. Here, the calculated value of $F w_{i j}$ may not be within $[0,1]$; thus, $F w_{i j}$ should be normalized by

$$
F w_{i j}^{\prime}=\frac{F w_{i j}}{\max \left(F w_{i j}\right)}
$$

For the informal collaborative relationship, it is mainly reflected in the personal social relationship among individuals. In the social network theory, for the measurement of node's social relationship influence, the common indexes include degree centrality, closeness centrality, and betweenness centrality [37]. Among them, degree centrality is used to depict the direct influence of node on its neighbor nodes [9]. However, it is the local measurement of node influence that can hardly reflect the global influence in the whole network. Closeness centrality is used to measure the influence of node on other nodes via network, which reflects the indirect influence of node [33]. Betweenness centrality balances the direct and indirect influences of node, which has very strong realistic meanings. Betweenness centrality is a global influence index, and it reflects the significance of node's location in the whole network and depicts the influence of node on information flow in network [4]. For the scenario of this work, the collaboration relationship among KCT partners is always with the information and knowledge flow. Based on the above analysis, betweenness centrality is

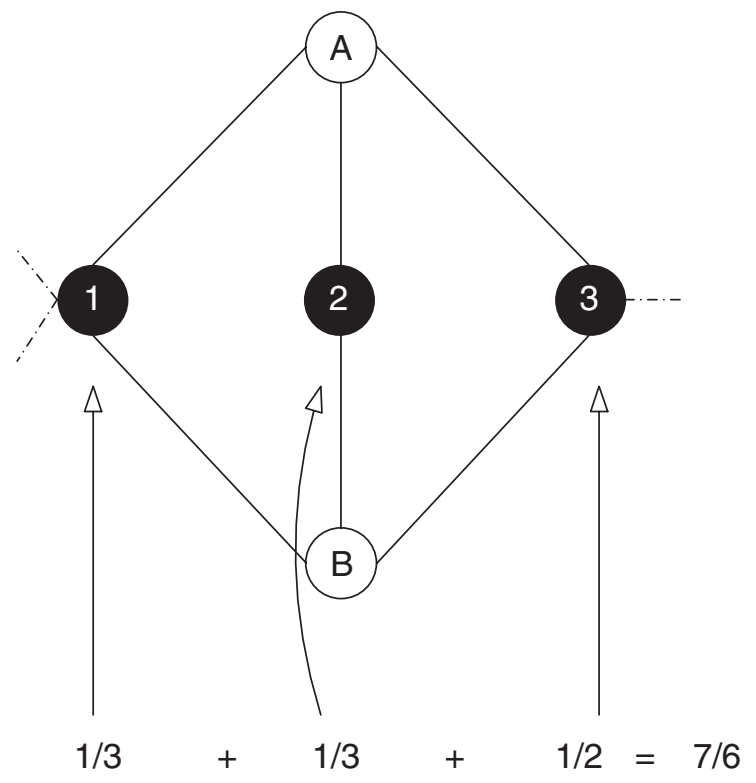

Figure 3: Partners A and B have Cooperated Three Projects Together, Labeled as 1, 2, and 3, Which had Four, Four, and Three Authors, Respectively.

The tie between $A$ and $B$ accordingly accrues weights $1 / 3,1 / 3$, and $1 / 2$ from the three projects for a total weight of $7 / 6$. 
a much more appropriate index to measure the node influence in this paper. Therefore, we use betweenness centrality as the node influence measurement index and define it as the informal collaboration influence of candidate. For the collaboration network $G$, the informal collaboration influence of candidate $P_{i}$ can be obtained using Eq. (6):

$$
B_{i}=\sum_{s \neq i \neq t \in G} \frac{\phi_{s t}(i)}{\phi_{s t}}
$$

where $\phi_{s t}$ is the number of shortest paths from candidate $P_{s}$ to $P_{t}$, and $\phi_{s t}(i)$ is the number of shortest paths from $P_{s}$ to $P_{t}$ that pass through $P_{i}$. Researches showed that tie weight has a correlation with the influence of nodes at each end $[1,30]$ and can be expressed as $w_{i j} \sim\left(k_{i} k_{j}\right)^{\theta}$, where $k_{i}$ and $k_{j}$ are the influence of nodes at each end and $\theta$ is a constant of specific weighted network. In this work, the strength of informal collaboration performance among candidates $I w_{i j}$ can be obtained using the above research results.

$$
I w_{i j}=\sqrt{B_{i} \cdot B_{j}}
$$

Similarly, the values of $I w_{i j}$ may not be within [0,1]; thus, $I w_{i j}$ can also be normalized by

$$
I w_{i j}^{\prime}=\frac{I w_{i j}}{\max \left(I w_{i j}\right)}
$$

By synthesizing the formal and informal collaborative performance, collaborative performance among candidates $P_{i}$ and $P_{j}$, that is, the tie weight of candidates' collaboration, can be obtained using

$$
w_{i j}=C P_{i j}=\mu \times F w_{i j}^{\prime}+v \times I w_{i j}^{\prime}
$$

where $\mu$ and $v$ are the weights to measure the formal and informal collaborative performance, respectively, and $\mu+v=1$.

\subsection{Model of Partner Selection Based on Knowledge and Collaboration Performance}

In this work, considering both individual knowledge competence and collaborative performance between a pair of candidates, the optimization model is built to select the desired $m$ partners from $n$ candidates.

$$
\begin{gathered}
\text { Max } \quad Z_{1}=\sum_{i=1}^{n} K C_{i} \cdot x_{i} \\
\operatorname{Max} \quad Z_{2}=\sum_{\substack { i=1 \\
\begin{subarray}{c}{j \neq 1 \\
j \neq i{ i = 1 \\
\begin{subarray} { c } { j \neq 1 \\
j \neq i } }\end{subarray}}^{n} C P_{i j} \cdot x_{i} x_{j} \\
\text { s.t. } \quad \sum_{i=1}^{n} x_{i}=m \\
x_{i}=\left\{\begin{array}{lr}
1 & \text { if candidate } P_{i} \text { is selected } \\
0 & \text { otherwise }
\end{array}\right.
\end{gathered}
$$

For models (10) to (13), it is a multiobjective 0-1 quadratic programming model. It is similar to the quadratic 0-1 model proposed by Kuo et al. [31] to study the maximum diversity problem, which has been proven as an NP-hard problem. 


\section{MOGA}

Partner selection for KCT is an NP-hard problem of multiobjective optimization. By reviewing the literature on partner selection and similar decision problem, we find that a variety of algorithms are used to solve the NP-hard models. These algorithms can be divided into two categories: the classical optimization methods (i.e. min-max approach [5] and weighted sum method [20]) and the intelligent algorithms (i.e. heuristic [18], simulated annealing [2], GA [41, 43], and particle swarm optimization (PSO) [47]). These examples show that the above algorithms are applicable to deal with partner selection problems to some extent. However, for the classical optimization methods, they can only get one particular optimal solution at one time, and the multiple Pareto-optimal solutions can hardly be found at each simulation run. As for the other intelligent algorithms, the main strategy of most of them is to convert a multiobjective problem into single objective problem by introducing a utility function or assigning weights to each objective, which will lead to the multiobjective optimization losing much of significance. To overcome the above shortcomings, we develop an MOGA to solve the multiobjective partner selection model of KCT in this work. Researches have shown MOGA is quite suitable to solve the NP-hard multiobjective problem and effective to solve partner selection problems in many fields [26, 45]. The developed MOGA is described as follows.

\subsection{Coding and Initialization}

Binary code is used to denote an individual as $[1,0,0, \ldots, 1,0]$ of $n$ codes, where 1 or 0 represents whether a candidate is chosen or not. For the proposed models (10) to (13), $m$ partners need to be selected from $n$ candidates, so there are $m$ codes encoded as 1 . Using this coding rule, individuals are randomly generated for initialization.

\subsection{Fitness Function}

In this work, a fast nondominated sorting approach is adopted to obtain the fitness value of individuals based on the work of Deb et al. [13]. First, every two individuals are selected, and their fitness values are compared by the dominating relationships and crowding distances. Second, all the individuals are divided into the nondominated fronts. The first nondominated front is marked as $F_{1}$ and the second nondominated front is marked as $F_{2}$ and so on. The individuals in former fronts are better than the ones in latter fronts, and this approach is called as nondominated sorting. Furthermore, the crowding distance is introduced then. An individual with small crowding distance implies that many other individuals are around it in the current population. In this way, the individuals in the same front can be sorted again by their crowding distances. Specifically, the individuals with larger crowding distance are better ones. The method to obtain crowding distances is presented as follows.

First, compute the gene distance between individuals. Suppose two individuals are $y_{i}=\left[y_{i 1}, y_{i 2}, \ldots, y_{i n}\right]$ and $y_{j}=\left[y_{j 1}, y_{j 2}, \ldots, y_{j n}\right]$, respectively. Thus, the gene distance $d_{i j}$ between the two individuals can be obtained using

$$
d_{i j}=\sum_{k=1}^{n}\left|y_{i k}-y_{j k}\right|
$$

Second, compare the gene distances. The smallest gene distance between individual $y_{i}$ and other individuals is made as crowding distance of individual $y_{i}$.

According to the dominating relationships and crowding distances, all the individuals are evaluated and given the fitness values by their evaluation results. 


\subsection{Selection Strategy}

For the selection strategy, the tournament selection method is adopted. First, select $r$ individuals randomly from the population, generally $r=2$. Then, select the best one from the $r$ individuals to survive to the next generation. Repeat the above steps to obtain the new population. It should be noted that the tournament selection uses the relative fitness values as the selection standard, which can avoid the influence from superindividual and reduce the risk of premature convergence to a certain degree.

\subsection{Crossover Operation}

Two-point crossover is adopted. However, for the proposed model, this crossover operator may generate infeasible solutions that do not satisfy the constraint (12).

For example, if $n=1$ and $m=5$, the two parents are denoted as

$$
\begin{aligned}
\text { Parent } 1 & =[0,1,1,0,0,1,1,0,0,1,0] \\
\text { Parent 2 } & =[1,0,0,1,1,0,1,0,0,1,0]
\end{aligned}
$$

If the crossover points are randomly selected as the fourth and seventh points, the offspring are represented as

$$
\begin{aligned}
& \text { Offspring } 1=[0,1,1,|1,1,0,1,| 0,0,1,0] \\
& \text { Offspring } 2=[1,0,0,|0,0,1,1,| 0,0,1,0]
\end{aligned}
$$

Obviously, the two offspring are infeasible solutions, which do not satisfy the constraint $m=5$. Hereby, a reparation strategy for this model is designed to repair the infeasible offspring. Specifically, for the infeasible offspring, the number of genes encoded as 1 should be increased or decreased until it satisfies the constraint (12). Meanwhile, the genes encoded as 0 are modified correspondingly.

\subsection{Mutation Operation}

The inversion operator is used to the individual. In this method, two reversal points in the individual are randomly selected, and then the genes between the two reversal points are reversed the order. Obviously, the inversion operator only changes the order of the genes but cannot increase or decrease the number of the genes with value of 1 . Thus, all the generated solutions satisfy constraint (12), and no infeasible solutions are produced.

\section{Illustrative Case}

In this section, we present a real case on partner selection for a KCT to illustrate the applicability of the proposed method in this paper.

$X M$ is one of the most creative mobile Internet companies in China, focusing on the development of intelligent electronic productions. It has made great success in designing, manufacturing, and developing smartphones and other smart products. To integrate effectively the internal and external knowledge resource, $X M$ takes collaborative knowledge innovation as an important strategy to hold its core competence in NPD. Now, the scale of KCTs in XM had been more than 1800 people, which are from various teams, such as Mobile Phone Hardware, System Development, Cloud Service, and Smart Products. 
To carry out a smartphone system development project, several KCTs are formed. Taking one of the KCTs as an example, its main objective is to improve the experience of user interface (UI). The candidates of this team are from different organizations and with multidiscipline knowledge. Seven desired partners need to be selected from 24 candidates. The decision-makers adopt four individual knowledge competence criteria to obtain the node weight as shown in Table 1 . Herein, $R_{1}, R_{2}$, and $R_{3}$ are objective criteria whose data are obtained from the knowledge collaborative system. $R_{4}$ is subjective whose values are obtained by expert assessment using scores from 1 to 9 ( 1 = very bad, $9=$ very good). The weights of $R_{1}, R_{2}, R_{3}$, and $R_{4}$ are obtained as $W=(0.30$, $0.20,0.30,0.20)$ by AHP. The original data and the node weights of candidates are shown in Table 2.

For the collaborative tie among candidates, it is determined by candidates' project cooperation information and data. The original information and data about the collaborative relationship among candidates can be obtained from the knowledge collaborative system of XM. However, there are so much data that they can hardly be listed in this paper. Using the tie weighted method proposed in Section 3.2.2, the tie weights are obtained, and the weighted social network of candidates is generated by the UCINET [3] (Figure 4).

Based on models (10) to (13), the model of partner selection for KCT in the real case can be built as follows:

$$
\begin{array}{ll}
\text { Max } & Z_{1}=0.637 x_{1}+0.770 x_{2}+0.288 x_{3}+0.491 x_{4}+\cdots+0.935 x_{22}+0.893 x_{23}+0.543 x_{24} \\
\text { Max } & Z_{2}=0.536 x_{1} x_{2}+0.510 x_{1} x_{4}+0.710 x_{1} x_{7} \cdots+0.549 x_{24} x_{17}+0.356 x_{24} x_{23} \\
\text { s.t. } & \sum_{i=1}^{24} x_{i}=7 \\
& x_{i}=1 \text { or } 0 \\
& i=1,2,3 \cdots, 24
\end{array}
$$

The MOGA developed in Section 4 is implemented to solve the above model. The parameters are set as Popsize $=100$, Maxgen $=200, p_{c}=0.95$, and $p_{m}=0.05$, where Popsize is the population size, Maxgen is the

\begin{tabular}{|c|c|c|c|c|c|c|c|c|c|c|c|}
\hline \multirow[t]{2}{*}{ Candidate } & \multicolumn{4}{|c|}{ Criteria } & \multirow[t]{2}{*}{ Weight } & \multirow[t]{2}{*}{ Candidate } & \multicolumn{4}{|c|}{ Criteria } & \multirow[t]{2}{*}{ Weight } \\
\hline & $R_{1}$ & $R_{2}$ & $R_{3}$ & $R_{4}$ & & & $R_{1}$ & $R_{2}$ & $R_{3}$ & $R_{4}$ & \\
\hline$P_{1}$ & 7 & 6 & 21 & 7 & 0.637 & $P_{13}$ & 6 & 3 & 18 & 4 & 0.190 \\
\hline$P_{2}$ & 11 & 7 & 23 & 6 & 0.770 & $P_{14}$ & 15 & 7 & 14 & 5 & 0.587 \\
\hline$P_{3}$ & 4 & 4 & 17 & 5 & 0.288 & $P_{15}$ & 12 & 5 & 18 & 6 & 0.588 \\
\hline$P_{4}$ & 5 & 6 & 19 & 6 & 0.491 & $P_{16}$ & 15 & 6 & 21 & 9 & 0.922 \\
\hline$P_{5}$ & 12 & 7 & 22 & 8 & 0.868 & $P_{17}$ & 13 & 7 & 20 & 7 & 0.791 \\
\hline$P_{6}$ & 8 & 5 & 16 & 4 & 0.345 & $P_{18}$ & 11 & 5 & 17 & 7 & 0.590 \\
\hline$P_{7}$ & 8 & 4 & 18 & 6 & 0.455 & $P_{19}$ & 7 & 6 & 24 & 9 & 0.812 \\
\hline$P_{8}$ & 3 & 5 & 17 & 5 & 0.305 & $P_{20}$ & 10 & 6 & 16 & 8 & 0.632 \\
\hline$P_{9}$ & 14 & 8 & 19 & 7 & 0.829 & $P_{21}$ & 9 & 5 & 12 & 5 & 0.268 \\
\hline$P_{10}$ & 6 & 5 & 23 & 8 & 0.674 & $P_{22}$ & 15 & 7 & 21 & 8 & 0.935 \\
\hline$P_{11}$ & 10 & 6 & 17 & 8 & 0.657 & $P_{23}$ & 12 & 7 & 23 & 8 & 0.893 \\
\hline$P_{12}$ & 9 & 4 & 20 & 7 & 0.578 & $P_{24}$ & 9 & 5 & 17 & 7 & 0.543 \\
\hline
\end{tabular}
number of generation, $p_{c}$ is the crossover probability, and $p_{m}$ is the mutation probability. The MOGA is coded

Table 1: Criteria for Individual Knowledge Competence

\begin{tabular}{lll}
\hline Objective & Criteria & Descriptions \\
\hline Individual & Publications $\left(R_{1}\right)$ & Quantity of publications related to the project \\
knowledge & Experience $\left(R_{2}\right)$ & Years in the work domain \\
competence & Knowledge capability $\left(R_{3}\right)$ & Total number of problems solved by cooperating with other members \\
& Know-who knowledge $\left(R_{4}\right)$ & Ability to get others' knowledge help \\
\hline
\end{tabular}

Table 2: Original Data and Node Weights of Candidates 


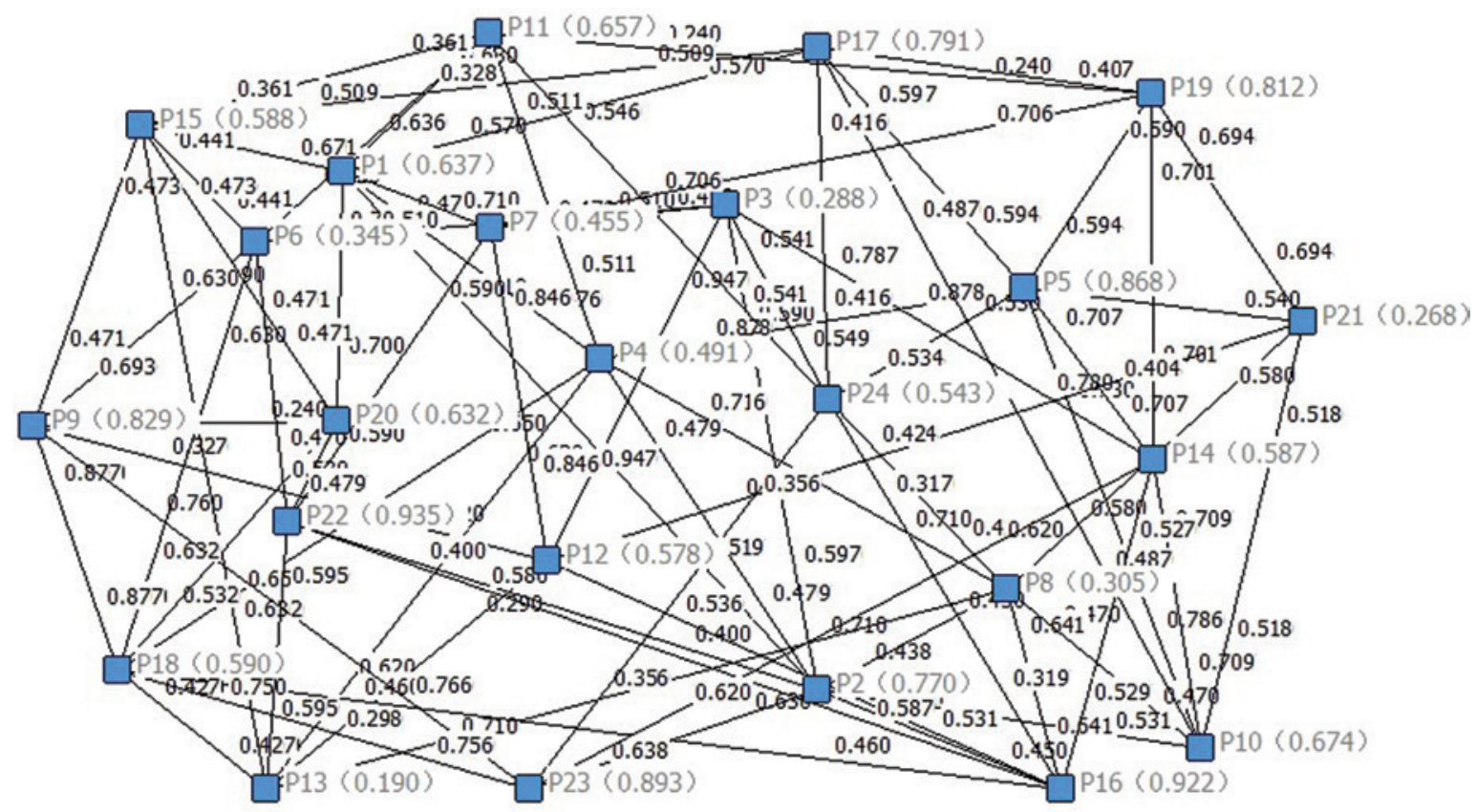

Figure 4: Weighted Social Network of Candidates.

by the MATLAB R2010a and run on a PC with an Intel Core i3-2330M 2.20 GHz CPU and 4 GB RAM. The MOGA is run 10 times, and the average runtime is $1.0516 \mathrm{~s}$. The Pareto-optimal solutions are listed in Table 3 and graphically shown in Figure 5. $Z_{1}^{*}$ and $Z_{2}^{*}$ are the Pareto-optimal values of the first and second objectives, respectively. These solutions are subsequently used to support the project managers and decision-makers to choose the desired KCT partners that best fit their requirements of individual knowledge competence or collaborative performance.

Table 3: Pareto-Optimal Solutions of Illustrative Case.

\begin{tabular}{lrr}
\hline Pareto-optimal solutions & & Optimal objectives \\
\cline { 2 - 3 } & $Z_{1}^{*}$ & $Z_{2}^{*}$ \\
\hline$(0,0,0,1,0,0,1,0,0,0,0,1,0,0,1,0,0,1,0,0,1,0,0,1)$ & 3.514 & 9.814 \\
$(1,0,0,1,0,0,1,1,0,0,0,1,0,0,0,0,0,1,0,0,0,0,0,1)$ & 11.161 \\
$(0,1,0,0,0,1,1,0,0,0,0,1,0,0,0,1,0,0,1,0,0,1,0,1)$ & 3.600 & 10.265 \\
$(1,0,1,0,0,0,1,0,1,0,0,1,0,1,0,0,0,1,0,0,0,0,0,0)$ & 3.773 & 10.982 \\
$(0,1,0,0,0,1,0,0,0,1,1,0,0,0,1,0,0,1,0,1,0,0,0,1)$ & 3.965 & 11.773 \\
$(0,0,0,1,0,0,0,1,1,0,0,0,0,0,0,0,0,1,1,0,1,0,1,0)$ & 11.201 \\
$(0,0,0,0,1,0,0,0,0,1,1,0,1,0,0,0,1,0,0,1,0,0,1,0)$ & 4.209 & 12.003 \\
$(1,1,0,0,0,0,0,0,1,0,1,0,0,1,0,0,1,0,0,1,0,0,0,0)$ & 4.457 & 11.986 \\
$(0,1,0,0,1,0,0,0,1,0,1,0,0,0,0,1,0,1,0,1,0,0,0,0)$ & 11.467 \\
$(0,0,0,0,1,0,0,0,1,0,1,0,0,0,1,1,0,0,0,1,0,0,1,0)$ & 4.703 & 12.479 \\
$(0,0,0,0,1,0,0,0,1,0,1,0,0,1,0,0,1,0,1,0,0,1,0,0)$ & 11.842 \\
$(0,0,0,0,0,0,0,0,0,1,0,0,0,0,0,1,1,0,1,1,0,1,1,0)$ & 5.266 & 12.186 \\
$(1,0,0,0,1,0,0,0,1,0,0,0,0,1,0,1,0,0,0,0,0,1,1,0)$ & 5.398 & 13.231 \\
$(0,1,0,0,1,0,0,0,1,0,0,0,0,0,0,1,0,0,1,1,0,1,1,0)$ & 5.478 & 13.032 \\
$(1,0,0,0,0,0,0,0,1,0,0,0,0,0,0,1,1,0,1,0,0,1,1,0)$ & 5.616 & 13.761 \\
$(0,1,0,0,1,0,0,0,0,1,0,0,0,0,0,1,1,0,1,0,0,1,0,0)$ & 5.670 & 13.489 \\
$(0,1,0,0,1,0,0,0,0,1,0,0,0,0,0,1,1,0,0,0,0,1,1,0)$ & 5.767 & 13.663 \\
$(0,0,0,0,1,0,0,0,1,0,0,0,0,0,0,1,1,0,1,0,0,1,1,0)$ & 5.819 & 13.546 \\
\hline & 5.926 & 6.007 \\
\end{tabular}




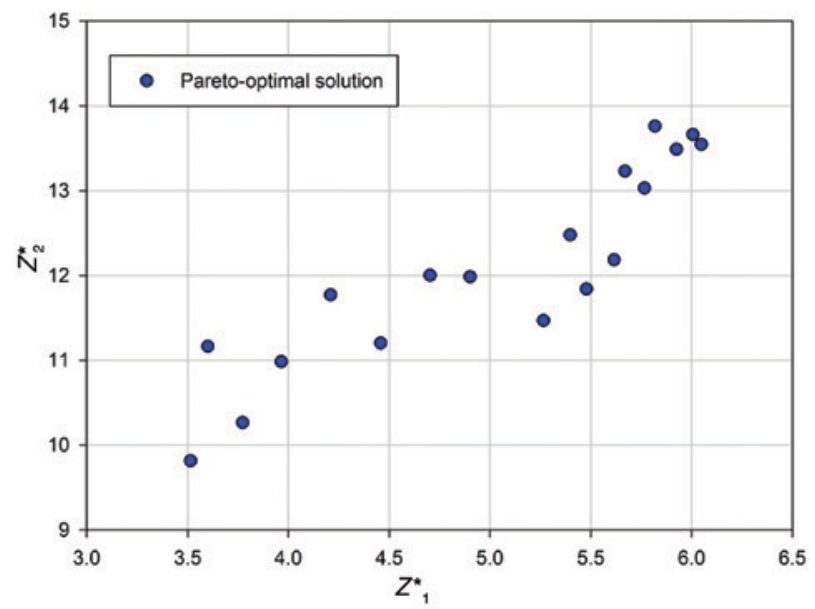

Figure 5: Pareto-Optimal Solutions for the Real Case.

To further verify the efficiency of the proposed MOGA, some simulation examples are given in this paper. In the simulation examples, the individual knowledge competence and collaboration performance among candidates are given with random numbers from 0 to 1 . The example parameters and results are shown in Table 4.

In Table 4, we can see that the solution space increases exponentially when $n$ and $m$ increase. Even for the example 5 with $O\left(100^{15}\right)$ solution space, the MOGA can run fast, whereas the traditional optimization methods are not competent to deal with it. In Table 4, we can also see that the runtime of MOGA is mainly determined by the parameters of $n$ and $m$. When the two parameters increase, the runtime will be longer accordingly. The results of simulation examples indicate that the proposed MOGA in this work can solve the large-scale partner selection problems efficiently. Also, this method can also be used as an algorithm base to solve the other similar decision problem, with modifications according to the specific decision problems.

\section{Conclusions}

Under the changing and uncertain competitive environments, the challenges of gaining sustainable development advantage have promoted the trend of collaborative knowledge innovation in firms. In this scenario, it is primary to assemble an effective and cooperative KCT with desired partners. Based on the weighted SNA, this paper presents a new method to solve the partner selection problem of KCT using the candidates' knowledge competence and collaboration performance. The weighted social network model of candidates is presented with the candidates as nodes and their collaboration relationship as edges. Moreover, the weight added methods of node weight and tie weight are proposed using the SNA approach. Based on the weighted social network of candidates, a multiobjective 0-1 model is built to select the desired partners, which is an NP-hard problem. An effective MOGA is then developed to solve the model. Finally, the proposed partner

Table 4: Simulation Example.

\begin{tabular}{lrrrrrrrl}
\hline Example & $\boldsymbol{n}$ & $\boldsymbol{m}$ & Popsize & Maxgen & $\boldsymbol{p}_{\mathrm{c}}$ & $\boldsymbol{p}_{\mathrm{m}}$ & Runtime & Solution space \\
\hline 1 & 20 & 5 & 100 & 200 & 0.95 & 0.05 & $0.9126 \mathrm{~s}$ & 252 \\
2 & 50 & 5 & 100 & 200 & 0.95 & 0.05 & $6.2863 \mathrm{~s}$ & $O\left(50^{5}\right)$ \\
3 & 100 & 5 & 100 & 200 & 0.95 & 0.05 & $24.3265 \mathrm{~S}$ & $O\left(100^{5}\right)$ \\
4 & 100 & 10 & 100 & 200 & 0.95 & 0.05 & $25.3784 \mathrm{~S}$ & $O\left(100^{10}\right)$ \\
5 & 100 & 15 & 100 & 200 & 0.95 & 0.05 & $27.4925 \mathrm{~S}$ & $O\left(100^{15}\right)$ \\
\hline
\end{tabular}


selection model and MOGA are applied to illustrate their application in a real case. The results indicated that the proposed decision method in this work is applicable and valid to deal with the partner selection problem of KCTs.

\subsection{Implications}

The partner selection for KCTs is a vital decision problem in the practices of knowledge collaboration. An effective and valid approach for partner selection would be significant to improve the quality of decision-making and warrant the future research. For the theoretical contributions, this study proposes a new weighted SNA method to comprehensively consider the individual knowledge competence and collaboration performance together of partner selection for KCTs. It overcomes the limitations of the existing researches relying on either the individual performance or the collaborative performance. Second, the proposed multiobjective partner selection model can be used as a basic model to study the related issues and more complex decision problems by modifying it according to the specific scenarios. Finally, the developed MOGA can obtain a Pareto-optimal solution set. Also, the simulation examples show that the MOGA are competent to deal with the large-scale partner selection problems.

For the decision-makers and managers of KCTs, the study can be a useful support for making effective decisions to select desired partners with comprehensive advantages and improving the collaboration performance of KCTs. This paper comprehensively considers the individual knowledge competence and the collaborative performance together. Based on this perspective, decision-makers are encouraged and supported to select desired partners who combine both individual knowledge competence and collaborative performance, which could be more likely to motivate the synergy among partners. In addition, this paper applied the SNA method to obtain and deal with the individual and collaborative information of candidates, which provide decision-makers with an effective approach to make better decisions in the condition of poor information. Moreover, the developed MOGA can be made as an effective tool to solve the multiobjective partner selection model and can provide decision-makers with a Pareto-optimal solution set. Therefore, decision-makers can make better partner selection decisions according to the project or task preferences on the individual or collaborative performance. In this way, it can help decision-makers to select desired partners to form KCTs that have more collaborative advantages.

\subsection{Limitations and Future Research}

Although this study improves our understanding of partner selection problem, its limitations should be also recognized, and future research would be definitely called for. First, for the more complicated partner selection problems, some more attributions should be considered in the decision process, such as the personality of partners, the knowledge complementarity among partners, and the investment of time and energy. Future work will integrate more attributions to solve the more complex issues and to make more effective and reliable decisions. Second, in the KCT formation process, the optimal size of the KCT is another important concern of decision-makers. Therefore, in future research, we will try to investigate the optimal size of the KCTs and combine it with this current study. In this way, it can be anticipated to obtain some more interesting results in this direction. Finally, this study focuses on the specific partner selection problem in KCT formation. However, it can also provide a new perspective for related issues with other backgrounds, i.e. crossfunctional team, research and development team, and concurrent engineering team. In the future, we will attempt to extend the proposed method to the above types of teams.

Acknowledgments: The authors are grateful for the support of the National Science Foundation of China (NSFC; Project No. 71571023) and the Chongqing Graduate Student Research Innovation Project (Project No. CYB15018). 


\section{Bibliography}

[1] A. Barrat, M. Barthelemy, R. Pastor-Satorras and A. Vespignani, The architecture of complex weighted networks, Proc. Natl. Acad. Sci. USA 101 (2004), 3747-3752.

[2] A. Baykasoglu, T. Das and S. Das, Project team selection using fuzzy optimization approach, Cyber. Syst. 38 (2007), 155-185.

[3] S. Borgatti, M. G. Everett, and L. C. Freeman, UCINET for Windows: software for social network analysis, Analytic Technologies, Harvard, MA, 2002.

[4] U. Brandes, S. P. Borgatti and L. C. Freeman, Maintaining the duality of closeness and betweenness centrality, Soc. Netw. 44 (2016), 153-159.

[5] C. Buchheim and J. Kurtz, Min-max-min robustness: a new approach to combinatorial optimization under uncertainty based on multiple solutions, Electron. Notes Discrete Math. 52 (2016), 45-52.

[6] R. Bunduchi, Trust, partner selection and innovation outcome in collaborative new product development, Prod. Plan. Control. 24 (2013), 1-13.

[7] U. Cantner and H. Graf, The network of innovators in Jena: an application of social network analysis, Res. Pol. 35 (2006), 463-480.

[8] S.-J. G. Chen and L. Lin, Modeling team member characteristics for the formation of a multifunctional team in concurrent engineering, IEEE. T. Eng. Manage. 51 (2004), 111-124.

[9] D.-B. Chen, H. Gao, L. Lü and T. Zhou, Identifying influential nodes in large-scale directed networks: the role of clustering, PLoS One 8 (2013), e77455.

[10] C.-F. Chien and L.-F. Chen, Data mining to improve personnel selection and enhance human capital: a case study in hightechnology industry, Expert. Syst. Appl. 34 (2008), 280-290.

[11] R. Cowan, N. Jonard and J. B. Zimmermann, Bilateral collaboration and the emergence of innovation networks, Manage. Sci. 53 (2007), 1051-1067.

[12] J. Crispim, N. Rego and J. P. D. Sousa, Stochastic partner selection for virtual enterprises: a chance-constrained approach, Int. J. Prod. Res. 53 (2015), 3661-3677.

[13] K. Deb, A. Pratap, S. Agarwal and T. Meyarivan, A fast and elitist multiobjective genetic algorithm: NSGA-II, IEEE. T. Evol. Comput. 6 (2002), 182-197.

[14] M. J. Donate and J. D. S. de Pablo, The role of knowledge-oriented leadership in knowledge management practices and innovation, J. Bus. Res. 68 (2015), 360-370.

[15] Z.-P. Fan, B. Feng, Z.-Z. Jiang and N. Fu, A method for member selection of R\&D teams using the individual and collaborative information, Expert. Syst. Appl. 36 (2009), 8313-8323.

[16] Z. Feng and B. Fan, A partner selection method for knowledge creation team based on collaborative effect, Chin. J. Manage. 2 (2012), 258-261.

[17] B. Feng, Z. Z. Jiang, Z. P. Fan and N. Fu, A method for member selection of cross-functional teams using the individual and collaborative performances, Eur. J. Oper. Res. 203 (2010), 652-661.

[18] E. L. Fitzpatrick and R. G. Askin, Forming effective worker teams with multi-functional skill requirements, Comput. Ind. Eng. 48 (2005), 593-608.

[19] P. A. Gloor, M. Paasivaara, D. Schoder and P. Willems, Finding collaborative innovation networks through correlating performance with social network structure, Int. J. Prod. Res. 46 (2008), 1357-1371.

[20] C. H. Goh, Y. C. A. Tung and C. H. Cheng, A revised weighted sum decision model for robot selection, Comput. Ind. Eng. 30 (1996), 193-199.

[21] R. Gulati, Alliances and networks, Strat. Manage. J. 19 (1998), 293-317.

[22] B. Haque, K. S. Pawar and R. J. Barson, Analysing organisational issues in concurrent new product development, Int. J. Prod. Econ. 67 (2000), 169-182.

[23] O. Hlaoittinun, E. Bonjour and M. Dulmet, A multidisciplinary team building method based on competency modelling in design project management, Int. J. Manage. Sci. Eng. Manage. 3 (2008), 163-175.

[24] M.-J. Huang, Y.-L. Tsou and S.-C. Lee, Integrating fuzzy data mining and fuzzy artificial neural networks for discovering implicit knowledge, Knowl. Based. Syst. 19 (2006), 396-403.

[25] L. Huang, Y. Lin, P. leromonachou, L. Zhou and J. Luo, Drivers and patterns of supply chain collaboration in the pharmaceutical industry: a case study on SMEs in China, Open J. Soc. Sci. 3 (2015), 23-29.

[26] W. Ip, M. Huang, K.-L. Yung and D. Wang, Genetic algorithm solution for a risk-based partner selection problem in a virtual enterprise, Comput. Oper. Res. 30 (2003), 213-231.

[27] L. B. Jeppesen and M. J. Molin, Consumers as co-developers: learning and innovation outside the firm, Technol. Anal. Strat. 15 (2003), 363-383.

[28] X. Jiang, X. L. Gu, Y. Ding and Y. Hu, Selection model of VGAgent from angle of collaboration, J. Wut 35 (2013), 144-148.

[29] T. Kaihara and S. Fujii, Game theoretic enterprise management in industrial collaborative networks with multi-agent systems, Int. J. Prod. Res. 46 (2008), 1297-1313. 
[30] M. Karsai, R. Juhász and F. Iglói, Nonequilibrium phase transitions and finite-size scaling in weighted scale-free networks, Phys. Rev. E 73 (2006), 036116.

[31] C. C. Kuo, F. Glover and K. S. Dhir, Analyzing and modeling the maximum diversity problem by zero-one programming, Decis. Sci. 24 (1993), 1171-1185.

[32] F. Li, Y. Yang, K. P. Yu, B. F. Bao and J. Z. Xie, Research on stability of customer collaborative product innovation system based on UWG, Stud. Sci. Sci. 32 (2014), 464-472.

[33] N. Magaia, A. P. Francisco, P. Pereira and M. Correia, Betweenness centrality in delay tolerant networks: a survey, Ad Hoc Netw. 33 (2015), 284-305.

[34] S. S. Msanjila and H. Afsarmanesh, Trust analysis and assessment in virtual organization breeding environments, Int. J. Prod. Res. 46 (2008), 1253-1295.

[35] A. Nagurney and Q. Qiang, A network efficiency measure with application to critical infrastructure networks, J. Global Optim. 40 (2008), 261-275.

[36] M. E. Newman, Scientific collaboration networks. II. Shortest paths, weighted networks, and centrality, Phys. Rev. E 64 (2001), 016132.

[37] C. S. Pereira and A. L. Soares, Improving the quality of collaboration requirements for information management through social networks analysis, Int. J. Inf. Manage. 27 (2007), 86-103.

[38] C. Raasch, C. Herstatt and P. Lock, The dynamics of user innovation: drivers and impediments of innovation activities, Int. J. Innov. Manage. 12 (2011), 377-398.

[39] P. B. Seybold, Outside innovation: how your customers will co-design your company’s future, Online 2 (2006), 62.

[40] W. Song, X. Ming and P. Wang, Collaborative product innovation network: status review, framework, and technology solutions, Concurr. Eng. 21 (2013), 55-64.

[41] J. Su, Y. Yang and X. Zhang, A member selection model of collaboration new product development teams considering knowledge and collaboration, J. Intell. Syst. 27 (2018), 213-229.

[42] X. Wang, B. Zhang and H. Chen, Member selection decision in VTIT based on multilevel extendible comprehensive evaluation, Sci. Res. Manage. 32 (2011), 108-112.

[43] H. Wi, S. Oh, J. Mun and M. Jung, A team formation model based on knowledge and collaboration, Expert. Syst. Appl. 36 (2009), 9121-9134.

[44] K. Yang and S. Wu, Information modeling of complex product based on multidisciplinary collaborative design, WIT. Trans. Model. Simul. 60 (2014), 769-776.

[45] W.-C. Yeh and M.-C. Chuang, Using multi-objective genetic algorithm for partner selection in green supply chain problems, Expert. Syst. Appl. 38 (2011), 4244-4253.

[46] K. P. Yoon and C.-L. Hwang, Multiple attribute decision making: an introduction, vol. 104, Sage Publications, Thousand Oaks, CA, 1995.

[47] L. Zhang and X. Zhang, Multi-objective team formation optimization for new product development, Comput. Ind. Eng. 64 (2013), 804-811.

[48] X. Z. Zhang, S. L. Liu, Y. Xie and Y. F. Zou, Cross-organizational collaborative innovation knowledge development research based on the complementarity of knowledge, Sci. Technol. Prog. Pol. 33 (2016), 136-141.

[49] A. Zzkarian and A. Kusiak, Forming teams: an analytical approach, IIE Trans. 31 (1999), 85-97. 